\title{
CIP-CSLR Scheme for Condensation and Evaporation Calculations of Water Droplets*
}

\author{
Ryo ONISHI**, Takeshi SUGIMURA** and Keiko TAKAHASHI** \\ ${ }^{* *}$ Earth Simulator Center, Japan Agency for Marine-Earth Science and Technology \\ 3173-25 Showa-machi, Kanazawa-ku Yokohama Kanagawa, 236-0001 Japan \\ E-mail:onishi.ryo@jamstec.go.jp
}

\begin{abstract}
We present a number-density advection based method designed to calculate the condensation and evaporation of water droplets. An Eulerian-in-radius discretization scheme is adopted, making the present model suitable for use in the spectral bin model for cloud microphysics. The advection equation is solved using the constrained interpolated profile-conservative Semi-Lagrangian with rational function (CIP-CLSR) scheme. In order to evaluate the present method based on the CIP-CSLR scheme, we have performed test calculations for a sequence of condensation and evaporation using the present method and conventional methods, and then compared the results. The comparisons have revealed the advantages of the present method in terms of numerical accuracy, numerical stability, conservation and computational cost.
\end{abstract}

Key words: Computational Method, Evaporation, Condensation, Spectral Bin Method, Advection Scheme

\section{Introduction}

Bulk cloud-microphysics models are widely used in mesoscale-cloud simulations. In those models, water substances are classified into several categories such as water vapor, cloud water, rain water, cloud ice, snow and graupel ${ }^{(1)(2)}$. The bulk models calculate cloud physical processes with some assumptions on droplet size distributions, for they cannot deal with droplet sizes explicitly. This sometimes limits the reliability of the bulk models for representing detailed processes in cloud physics. In contrast, spectral-bin models can explicitly calculate droplet sizes using several tens or hundreds of size bins (classes) ${ }^{(3)(4)}$. Although the spectral-bin models require larger computational costs, they are promising for detailed cloud simulations.

The spectral-bin models calculate size changes due to activations of cloud condensation nuclei $(\mathrm{CCN})$, phase changes, collisions and break-ups. Particularly, the calculation for phase changes is worth careful consideration in two points: One is that it is computationally expensive and requires efficient calculation. Another is that it influences the computational stability since phase changes influence dynamics through heat releases.

Droplet growth is treated as an advection of number densities between size bins. Then, we need to care the CFL restriction between the size resolution, $\Delta r$, and growth speed, $d r / d t$. The scheme which can relax the CFL restriction is, therefore, preferred for efficient calculation. The total number of droplets is conserved through condensation growth, while it is changed through collision growth. If the conservation is violated, it may make the cloud simulation unstable due to unrealistic heat releases. The conservative scheme is, therefore, preferred for computational stability.

The adoption of a Semi-Lagrangian scheme is one solution to relax the CFL restriction,

${ }^{*}$ Received 7 Aug., 2009 (No. T1-08-0271) Japanese Original : Trans. Jpn. Soc. Mech. Eng., Vol.74, No.748, B (2008), pp.2443-2451 (Received 31 Mar., 2008) [DOI: 10.1299/jee.5.1]

Copyright $\odot 2010$ by JSME 
whereas conventional Semi-Lagrangian schemes are not conservative. On the other hand, WAF (Weighed Averaged Flux) schemes ${ }^{(5)(6)}$, which are based on flux form, guarantee the conservation. However, they cannot avoid the CFL restriction since they are differential schemes. One solution that can achieve both relaxing the CFL restriction and guaranteeing the conservation is to adopt the CIP-CSLR (Constrained Interpolated Profile-Conservative Semi-Lagrangian with Rational function) scheme $^{(7)}$. The CIP-CSLR scheme is a Semi-Lagrangian scheme and can relax the CFL restriction. It also guarantees the conservation by considering the integration inside each control volume. However, the CIP-CSLR scheme has not been employed and validated for phase-change calculations in the spectral-bin model.

This study develops a phase-change calculation method based on the CIP-CSLR scheme for Eulerian-in-radius discretization, which is suitable for the spectral-bin model for cloud microphysics. Our developed method is evaluated through an idealized numerical experiment for condensing and evaporating water droplets in a humid air. The experiment is also performed with several conventional methods, which are based on first-order upwind scheme, third-order upwind scheme, first-order Semi-Lagrangian scheme, and WAF (Weighted Average Flux) scheme with SUPERBEE-flux limiter. Results from our developed method and those from the conventional methods are compared to investigate the relative features in terms of numerical diffusion, numerical accuracy, number conservation and computational cost.

\section{Symbols}

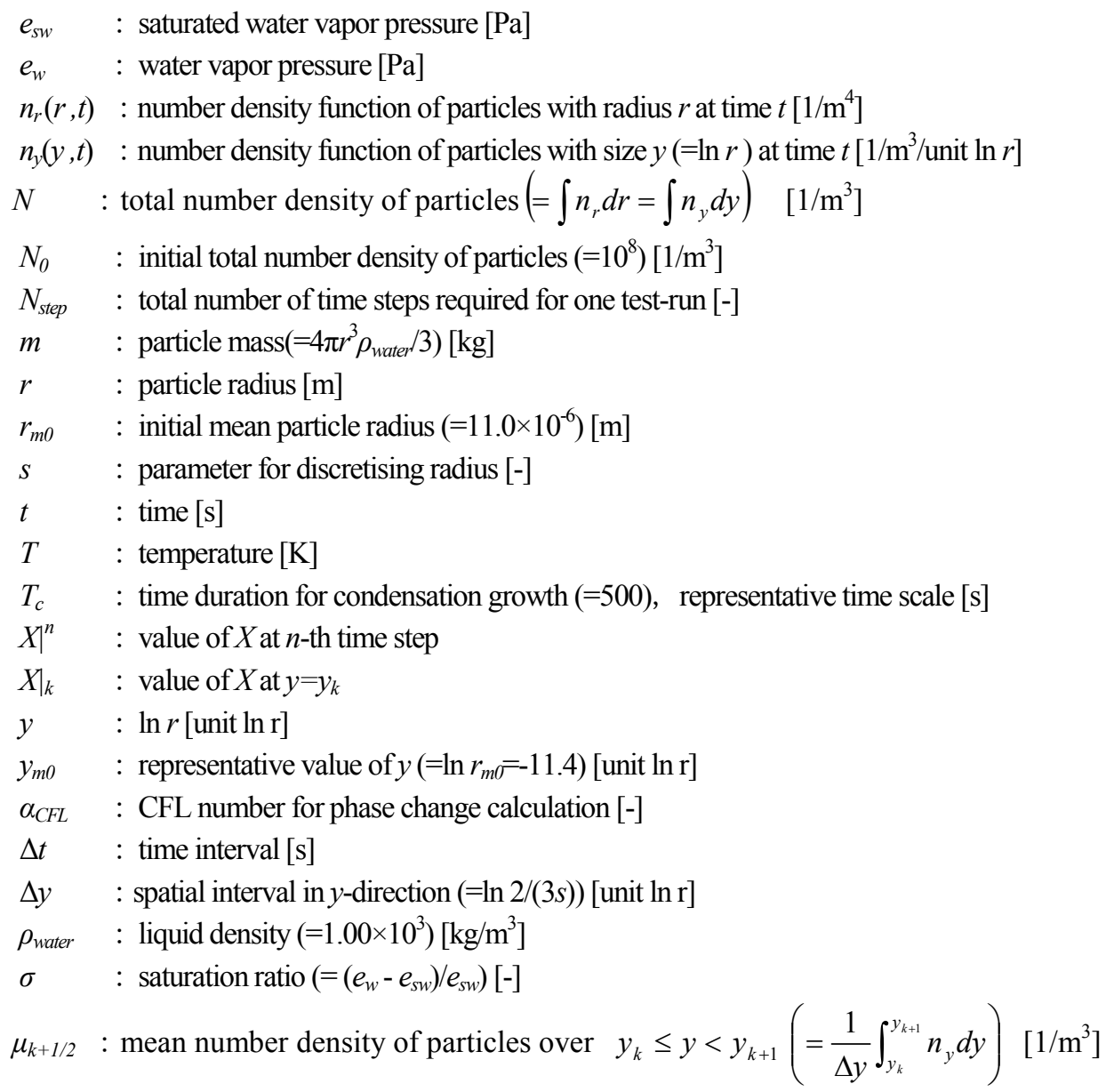




\section{Condensation and evaporation calculation method}

\subsection{Governing equations}

The spectral-bin model calculates the time evolution of particle number density function, $n_{r}(r, t)$. The term of the temporal change due to phase change is written as

$$
\left(\frac{\partial n_{r}(r, t)}{\partial t}\right)_{\text {cond }}=-\frac{\partial}{\partial r}\left[n_{r}(r, t) \frac{d r}{d t}\right]
$$

where $d r / d t$ is the growth rate in radius and calculated as ${ }^{(8)}$

$$
\frac{d r}{d t}=\frac{\sigma}{r \rho_{\text {water }}\left\{\left(5.58 \times 10^{10} / T^{2}\right)+\left(2.04 \times 10^{6} T / e_{s w}\right)\right\}} .
$$

The super saturation ratio, $\sigma\left(=\left(e_{w}-e_{s w}\right) / e_{s w}\right)$ is derived from the saturated water vapor pressure, $e_{s w}[\mathrm{~Pa}]$, fitted as

$$
e_{s w}=611.2 \exp \left\{17.67\left(\frac{T-273.15}{T-29.65}\right)\right\} \text {. }
$$

Since the growth rate is larger in magnitude when the droplet is smaller, the finer resolution is required for smaller sizes. The logarithmic form is, therefore, convenient for discretizing the radius ${ }^{(8)}$.

$$
y=\ln r .
$$

Then, the following number density function is used instead of $n_{r}(r, t)$;

$$
n_{y}(y, t) d y=n_{r}(r, t) d r .
$$

The relation of $d r / d y=r$ yields

$$
n_{y}(y, t)=r n_{r}(r, t)
$$

Then, Equation (1) is rewritten as

$$
\frac{\partial n_{y}(y, t)}{\partial t}=-\frac{\partial \lambda n_{y}(y, t)}{\partial y},
$$

where $\lambda$ is the growth rate in $y, d y / d t$. Equation (2) and $d y / d r=1 / r$ yield

$$
\begin{aligned}
\lambda & =\frac{\sigma}{\rho_{\text {water }}\left\{\left(\frac{5.58 \times 10^{10}}{T^{2}}\right)+\left(\frac{2.04 \times 10^{6} T}{e_{s w}}\right)\right\}} e^{-2 y}, \\
& \equiv A e^{-2 y}
\end{aligned}
$$

where the fraction in the right-hand-side is defined as $A$.

Equation (7) is based on a flux form (conservative form) and shows that the temporal change (time derivative) of $n_{y}$ is proportional to the spatial ( $y$-space) derivative of $\lambda n_{y}$. Expanding the right-hand-side of Equation (7) into advection and non-advection terms, we obtain the following non-conservative form; 


$$
\frac{\partial n_{y}}{\partial t}=-\lambda \frac{\partial n_{y}}{\partial y}-n_{y} \frac{\partial \lambda}{\partial y} .
$$

\subsection{Discretization of governing equations}

In order to numerically compute Equations (7) and (9), the equations and variables are to be discretized in space $(y)$ and time $(t)$.

Berry $(1967)^{(9)}$ discretized $y$ in equal ratio of mass as

$$
m_{k}=2^{1 / s} \times m_{k-1},
$$

where it is called mass doubling when $s=1$. This leads to

$$
y_{k}=y_{k-1}+\frac{\ln 2}{3 s} .
$$

This means $y_{k}$ is arithmetic series with equal difference of $\Delta y=\ln 2 /(3 s)$. In this study, first-order Euler scheme is adopted for time integration.

$$
\left.n_{y}\right|^{n+1}=\left.n_{y}\right|^{n}+\left.\frac{\partial n_{y}}{\partial t}\right|^{n} \Delta t,
$$

where (variable) $\left.\right|^{n}$ denotes the value of variable at $n$-th time step $\left(t^{n}\right)$.

\section{Advection schemes}

\subsection{CIP-CSLR scheme}

CIP-CSLR (constrained interpolated profile-conservative Semi-Lagrangian with rational function) scheme $^{(7)}$ is based on a Semi-Lagrangian scheme, and calculates the temporal change due to advection with trajectory calculation. The interpolation function at target grid as well as point values $\left.n_{y}\right|_{k}$, where (variable) $\left.\right|_{k}$ denotes the value of variable at $y=y_{k}$, is calculated. Adopting a rational function for the interpolation function prevents numerical oscillations. The conservation is guaranteed since the mean number density, i.e., integral of the number density, at $k$-th bin $\left(y_{k} \leq y<y_{k+1}\right), \mu_{k+1 / 2}=\frac{1}{\Delta y} \int_{y_{k}}^{y_{k+1}} n_{y} d y$, is calculated in a flux form. The governing equation of $\mu_{k+1 / 2}$ is derived by averaging the Equation (7) over a bin as

$$
\begin{aligned}
& \frac{1}{\Delta y} \int_{y_{k}}^{y_{k+1}} \frac{\partial n_{y}}{\partial t} d y=-\frac{1}{\Delta y} \int_{y_{k}}^{y_{k+1}} \frac{\partial\left(\lambda n_{y}\right)}{\partial y} d y \\
& \Leftrightarrow \frac{\partial \mu_{k+1 / 2}}{\partial t}=-\frac{\left(\lambda n_{y}\right)_{k+1}-\left(\lambda n_{y}\right)_{k}}{\Delta y} .
\end{aligned}
$$

The following procedure is our developed algorithm to solve Equation (9), that is, to obtain the number density at $y=y_{k}$ at $n+1$-th step, $\left.n_{y}\right|_{k} ^{n+1}$, from the values at $n$-th step:

PROCEDURE [1] Upstream point, $y_{k}^{+}$, of $y_{k}$ is calculated as 


$$
y_{k}^{+}=y_{k}-\int_{t^{n}}^{t^{n+1}} \lambda d t
$$

PROCEDURE [2]: Neighboring points $y_{l(k)}$ and $y_{l(k)+1}$ of $y_{k}{ }^{+}$are searched:

$$
y_{l(k)} \leq y_{k}^{+}<y_{l(k)+1} .
$$

PROCEDURE [3] The rational interpolation function, $f_{l(k)}(y)$, is prepared;

$$
f_{l(k)}(y)=\frac{a_{l}+2 b_{l}\left(y-y_{l}\right)+\beta_{l} b_{l}\left(y-y_{l}\right)^{2}}{\left[1+\beta_{l}\left(y-y_{l}\right)\right]^{2}} .
$$

The three variables, $a_{l}, b_{l}$ and $\beta_{l}$, in Equation (16) are determined from the values at the neighboring points, $\left.n_{y}\right|_{l(k)}$ and $\left.n_{y}\right|_{l(k)+1}$, and the mean number density, $\mu_{l(k)+1 / 2}$, as

$$
\left\{\begin{array}{l}
\frac{1}{\Delta y} \int_{y_{l(k)}}^{y_{l(k)+1}} f_{l(k)} d y=\mu_{l(k)+1 / 2} \\
f_{l(k)}\left(y_{l}\right)=\left.n_{y}\right|_{l(k)} \\
f_{l(k)}\left(y_{l(k)+1}\right)=\left.n_{y}\right|_{l(k)+1}
\end{array} .\right.
$$

PROCEDURE [4] The substitution of $y_{k}{ }^{+}$into the above interpolation function yields the upstream value, $\left.n_{y}\left(y_{k}^{+}\right)\right|^{n}$. This operation is equivalent to calculating the advection term in Equation (9). The intermediate value of number density function at $y_{k}$ at $n+1$-th step is written as $\left.\tilde{n}_{y}\right|_{k} ^{n+1}$, that is,

$$
\left.\tilde{n}_{y}\right|_{k} ^{n+1}=\left.n_{y}\left(y_{k}^{+}\right)\right|^{n}=f_{l(k)}\left(y_{k}^{+}\right) \text {. }
$$

PROCEDURE [5] In addition, we need to calculate the non-advection term in Equation (9). The non-advection term is calculated as

$$
\begin{aligned}
\left.n_{y}\right|_{k} ^{n+1} & =\left.\widetilde{n}_{y}\right|_{k} ^{n+1}-\int_{t^{n}}^{t^{n+1}} n_{y} \frac{\partial \lambda}{\partial y} d t \\
& =f_{l(k)}\left(y_{k}^{+}\right)+2 \int_{t^{t}}^{t^{n+1}} n_{y} \lambda d t, \\
& \approx f_{l(k)}\left(y_{k}^{+}\right)+2 g_{k}
\end{aligned}
$$

where $g_{k}$ is defined, with the aid of $y_{k}(t)=y_{k}-\int_{t}^{t^{n+1}} \lambda d t$, as

$g_{k}=\int_{t^{n}}^{t^{n+1}} \lambda\left(y_{k}(t)\right) f\left(y_{k}(t)\right) d t$.

This $g_{k}$ is interpreted as the flux of particle number density through $y=y_{k}$ between $t^{n}$ and $t^{n+1}$. Therefore, it is equivalent to the integral of particle number density between $y_{k}^{+}$ and $y_{k}$. Then, $g_{k}$ is obtained as 


$$
\begin{aligned}
g_{k} & =\int_{y_{k}^{+}}^{y_{k}} f(y) d y \\
& =\int_{y_{k}^{+}}^{y_{l(k)}} f(y) d y+\int_{y_{l(k)}}^{y_{k}} f(y) d y \\
& =-\frac{a_{l}\left(y_{k}^{+}-y_{l(k)}\right)+b_{l}\left(y_{k}^{+}-y_{l(k)}\right)^{2}}{1+\beta_{l}\left(y_{k}^{+}-y_{l(k)}\right)}+G_{k}
\end{aligned}
$$

where $G_{k}$ is defined depending on the sign of $\lambda$ as

$$
G_{k}=\left\{\begin{array}{ll}
\Delta y \times \sum_{i=l(k)-1}^{k-1} \mu_{i+1.2}^{n} & (\lambda \geq 0) \\
-\Delta y \times \sum_{i=k}^{l(k)-1} \mu_{i+1.2}^{n} & (\lambda<0)
\end{array} .\right.
$$

PROCEDURE [6] The mean number density at $k$-th bin at $n+1$ step, $\mu_{k+1 / 2}^{n+1}$, is obtained by integrating Equation (13) from $t^{n}$ to $t^{n+1}$ as

$$
\mu_{k+1 / 2}^{n+1}=\mu_{k+1 / 2}^{n}-\frac{g_{k+1}-g_{k}}{\Delta y} .
$$

These procedures from [1] to [6] calculate the point and mean values of particle number density at $n+1$-th step, $\left.n_{y}\right|_{k} ^{n+1}$ and $\mu_{k+1 / 2}^{n+1}$ from the values at $n$-th step.

\subsection{Conventional non-conservative schemes}

In this study, we pick up the first order upstream scheme, third order upstream scheme and first order Semi-Lagrangian scheme as conventional non-conservative advection schemes. These schemes solve Equation (9), which is written in a non-conservative form. The first order upstream scheme calculates the advection in Equation (9) as

$$
\begin{aligned}
\left(-\lambda \frac{\partial n_{y}}{\partial y}\right)_{k} & =\frac{1}{2} \frac{\left|\lambda_{k}\right|}{\Delta y}\left(\left.n_{y}\right|_{k+1}-\left.2 n_{y}\right|_{k}+\left.n_{y}\right|_{k-1}\right) . \\
& -\frac{1}{2} \frac{\lambda_{k}}{\Delta y}\left(\left.n_{y}\right|_{k+1}-\left.n_{y}\right|_{k-1}\right)
\end{aligned}
$$

The K-K scheme ${ }^{(10)}$ is one of the third order upstream schemes. The K-K scheme solves the advection as

$$
\begin{aligned}
\left(-\lambda \frac{\partial n_{y}}{\partial y}\right)_{k} & =-\frac{1}{4} \frac{\left|\lambda_{k}\right|}{\Delta y}\left(\left.n_{y}\right|_{k+2}-\left.4 n_{y}\right|_{k+1}+\left.6 n_{y}\right|_{k}-\left.4 n_{y}\right|_{k-1}+\left.n_{y}\right|_{k-2}\right) \\
& -\frac{1}{12} \frac{\lambda_{k}}{\Delta y}\left(-\left.n_{y}\right|_{k+2}+\left.8 n_{y}\right|_{k+1}-\left.8 n_{y}\right|_{k-1}+\left.n_{y}\right|_{k-2}\right)
\end{aligned} .
$$

In the first order Semi-Lagrangian scheme, the advection calculation is achieved by searching the upstream value as in Equation (14). The simple linear interpolation is used instead of the rational function used in the CIP-CSLR scheme.

The non-advection term in Equation (9) is analytically solved as 


$$
\left(-n_{y} \frac{\partial \lambda}{\partial y}\right)_{k}=\left.2 A n_{y}\right|_{k} e^{-2 y_{k}} .
$$

Summing the advection and non-advection terms yields the values at the next time step.

\subsection{Conventional conservative scheme}

This study employs the WAF (Weighted Average Flux) scheme ${ }^{(5)}$ with SUPERBEE-flux limiter for Equation (7) written in a conservative form (flux form). The scheme has second order accuracy in space and achieves the feature of TVD (Total Variation Diminishing) by the adoption of SUPERBEE-flux limiter ${ }^{(6)}$. In the WAF scheme, the right-hand-side of Equation (7) is calculated as

$$
\left(-\frac{\partial \lambda n_{y}}{\partial y}\right)_{k}=-\frac{\left(f_{k+1 / 2}-f_{k-1 / 2}\right)}{\Delta y},
$$

where $f$ is called the WAF flux. A TVD-type WAF flux, $f^{T V D}$, is written as

$$
\left\{\begin{array}{l}
f_{k+1 / 2}^{T V D}=\frac{1}{2}\left(1+\phi_{k+1 / 2}\right)\left(\left.\lambda_{k+1 / 2} n_{y}\right|_{k}\right)+\frac{1}{2}\left(1-\phi_{i+1 / 2}\right)\left(\left.\lambda_{k+1 / 2} n_{y}\right|_{k+1}\right) \\
f_{k-1 / 2}^{T V D}=\frac{1}{2}\left(1+\phi_{k-1 / 2}\right)\left(\left.\lambda_{k-1 / 2} n_{y}\right|_{k-1}\right)+\frac{1}{2}\left(1-\phi_{k-1 / 2}\right)\left(\left.\lambda_{k-1 / 2} n_{y}\right|_{k}\right)
\end{array},\right.
$$

where $\phi$ is called a flux limiter. One of the widely used flux limiters is the SUPERBEE-flux limiter, which is defined as

$$
\phi_{k+1 / 2}(p,|c|)=\left\{\begin{array}{lc}
1 & \left(p_{k+1 / 2} \leq 0\right) \\
1-2\left(1-\left|c_{k+1 / 2}\right|\right) p_{k+1 / 2} & \left(0 \leq p_{k+1 / 2} \leq 1 / 2\right) \\
\left|c_{k+1 / 2}\right| & \left(1 / 2 \leq p_{k+1 / 2} \leq 1\right) \\
1-\left(1-\left|c_{k+1 / 2}\right|\right) p_{k+1 / 2} & \left(1 \leq p_{k+1 / 2} \leq 2\right) \\
2\left|c_{k+1 / 2}\right|-1 & \left(2 \leq p_{k+1 / 2}\right)
\end{array},\right.
$$

where $c_{k+1 / 2}$ is a local CFL number defined as $\lambda_{k+1 / 2} \Delta t / \Delta y$, and $p_{k+1 / 2}$ is called flow parameter defined as

$$
p_{k+1 / 2}=\frac{\Delta_{\text {upwind }}}{\Delta_{\text {local }}}=\left\{\begin{array}{ll}
\frac{\left.n_{y}\right|_{k}-\left.n_{y}\right|_{k-1}}{\left.n_{y}\right|_{k+1}-\left.n_{y}\right|_{k}} & \left(\lambda_{k+1 / 2}>0\right) \\
\frac{\left.n_{y}\right|_{k+2}-\left.n_{y}\right|_{k+1}}{\left.n_{y}\right|_{k+1}-\left.n_{y}\right|_{k}} & \left(\lambda_{k+1 / 2}<0\right)
\end{array} .\right.
$$

\section{Scheme intercomparison}

\subsection{Numerical setups}

As an ideal test run, we carry out a sequence of condensation and evaporation of water droplets in a humid air parcel. At first, water droplets grow by condensation in saturated condition of $\sigma=\sigma_{c}(=0.001$, i.e. $0.1 \%)$ for $T_{c}(=500 \mathrm{~s})$. This supersaturation rate is a typical 
one in convective clouds. After the condensation, the water droplets evaporate under unsaturated condition of $\sigma_{e}=-0.01\left(=-10 \sigma_{c}\right)$ for $T_{e}=0.1 T_{c}$. For simplicity, we fix the air temperature at $293 \mathrm{~K}$ to concentrate on the phase change calculations. We set $\sigma_{c} T_{c}$ equivalent to $-\sigma_{e} T_{e}$, which means that the size distribution of droplets returns to the initial state after the sequence of condensation and evaporation processes.

The initial size distribution is set as the maritime cloud condensation nuclei in Tzivion et al. (1994) ${ }^{(11)}$;

$$
n_{r}(r)=a r^{\beta} \exp \left[-b r^{\gamma}\right]
$$

where $\beta=2, \gamma=1, a=1.01 \times 10^{8} \mathrm{~m}^{-6}$ and $b=2.73 \times 10^{5} \mathrm{~m}^{-1}$. Eventually, the mean radius of this distribution is $r_{m 0}=11.0 \times 10^{-6} \mathrm{~m}$ and initial particle number density is $N_{0}=10^{8} \mathrm{~m}^{-3}$.

\subsection{Numerical conditions}

Table 1 shows the numerical setup for spatial (y-direction) discretization. In mesoscale simulations, $s$ in equation (10) is often set as $s=1^{(12)}$. In this study, we perform the calculations with various $s$ including the case of $s=1$ in order to investigate the spatial accuracy. In addition, we perform an extremely high resolution calculation with $s=128$ in order to obtain a reference data (RUN-REF). In all calculations, the smallest and largest bin sizes are fixed; $r_{\min }=1.0 \times 10^{-6} \mathrm{~m}\left(y_{\min }=-13.8\right)$ and $r_{\max }=64.0 \times 10^{-6} \mathrm{~m}\left(y_{\max }=-9.66\right)$.

Table 2 shows the setup for temporal discretization. The CFL number for phase change calculation is represented by $\alpha_{C F L}$.

$$
\alpha_{C F L}=\left|\lambda_{k=1}\right| \frac{\Delta t}{\Delta y}
$$

The growth rate $\lambda$ reaches its maximum when the size is minimum, that is, the CFL is restricted by $\lambda_{k=1}$. In this study, four kinds of $\alpha_{C F L}$ are used to investigate the time accuracy of the schemes.

We need to select the spatial resolution from Table 1 and temporal resolution from Table 2. We refer, for example, the run with $s=4$ and $\alpha_{C F L}=0.5$ to "RUN-S4+CFL05" hereafter.

Table 1 Settings of spatial ( $y$-direction) discretizations.

\begin{tabular}{rcc}
\hline RUN & $s$ & $\Delta y /\left|y_{m 0}\right|$ \\
\hline \hline S1 & 1 & $2.02 \times 10^{-2}$ \\
\hline S2 & 2 & $1.02 \times 10^{-2}$ \\
\hline S4 & 4 & $5.06 \times 10^{-3}$ \\
\hline S8 & 8 & $2.53 \times 10^{-3}$ \\
\hline S16 & 16 & $1.26 \times 10^{-3}$ \\
\hline REF & 128 & $1.57 \times 10^{-4}$ \\
\hline
\end{tabular}

Table 2 Settings of temporal discretizations.

\begin{tabular}{rc}
\hline RUN & $\alpha_{\text {CFL }}$ \\
\hline \hline CFL01 & 0.1 \\
\hline CFL05 & 0.5 \\
\hline CFL2 & 2.0 \\
\hline CFL10 & 10.0 \\
\hline
\end{tabular}




\section{Results and Discussion}

\subsection{Time evolution of size distribution}

Figure 1 shows the particle size distribution at $t / T_{c}=0,0.5,1.0$ and 1.1 for RUN-REF+CFL01. The vertical axis shows the number density function normalized by $y_{m} 0$ and the initial number density $N_{0}$. After the sequence of condensation for $0<t / T_{c}<1.0$ and evaporation for $1.0<t / T_{c}<1.1$, the size distribution at $t / T_{c}=1.1$ should be consistent to the initial size distribution. The pair of results from the first-order upwind and first-order Semi-Lagrangian schemes, and another pair of results from the WAF and CIP-CSLR schemes are both identical. The WAF and CIP-CSLR schemes showed converged results at $s=64$ and 128(RUN-REF). Therefore, we hereafter use the averaged values between the WAF and CIP-CSLR results for RUN-REF as reference. Results of first-order upwind and first-order Semi-Lagrangian schemes do not agree with the reference, i.e., they do not converge even with the highest spatial resolution of $s=128$.

The sub-figure in Figure 1 focuses the range $-0.910<y / y_{m 0}<-0.905$ in order to magnify the tail of the distribution at $t / T_{c}=1.0$. Result of the third-order upwind scheme at $t / T_{c}=1.0$ shows an overshooting due to a numerical oscillation around $y=-0.907$. Other schemes do not show such an overshooting.

Figure 2(a) and 2(b) show the size distributions at (a) $t / T_{c}=1.0$ and (b) $t / T_{c}=1.1$ for RUN-S4+CFL05. In other words, Figure 2(a) shows the results after condensations and Figure 2(b) after evaporations. Figure 2(a) reveals that all the schemes produce smoother distributions with smaller peak values than the reference due to numerical diffusions. In particular, first-order schemes have large numerical diffusions and show less than one-thirds of the peak values than the reference. Furthermore, they show significant phase latencies. The third-order upwind scheme has less numerical diffusions than the first-order schemes, however suffers from the numerical oscillation. The CIP-CSLR scheme shows the best result in terms of the numerical diffusion and numerical oscillation. The WAF scheme shows the second best result. Figure 2(b) reconfirms the above features. The CIP-CSLR, WAF and third-order upwind schemes show better results in order. The CIP-CSLR scheme has similar peak value to the reference.

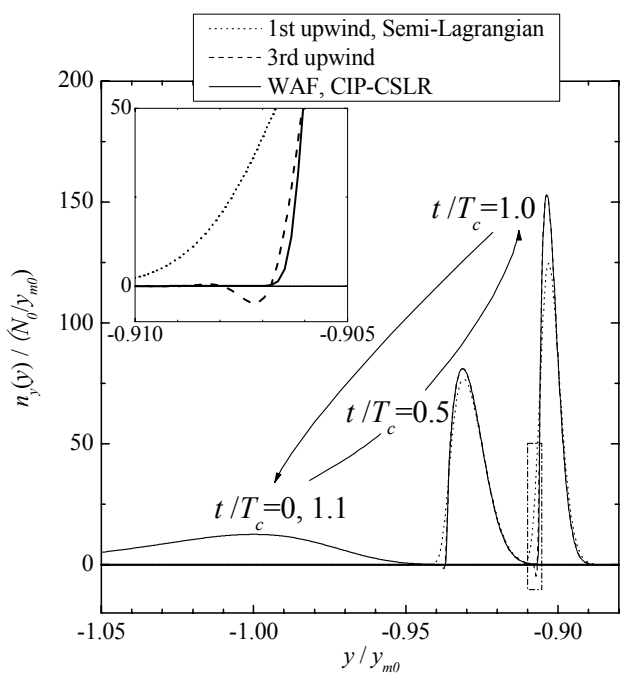

Fig. 1 Reference normalized particle number density functions, $n_{y} /\left(N_{0} / y_{m 0}\right)$, at $t / T_{c}=0,0.5$, 1.0 and 1.1, obtained from RUN-REF+CFL01. 


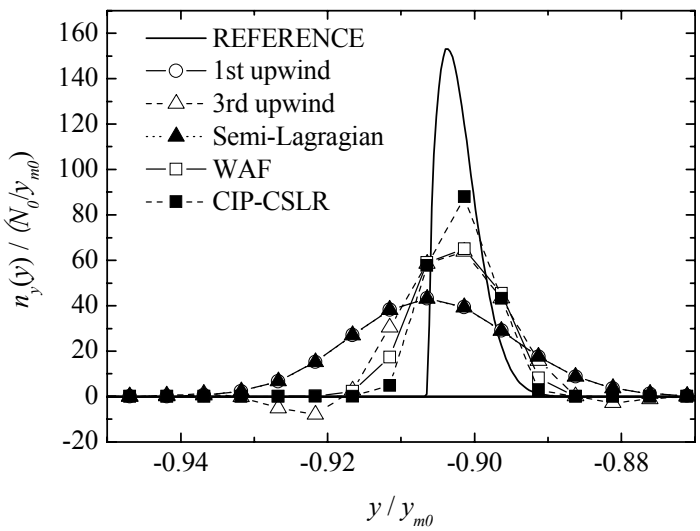

(a)

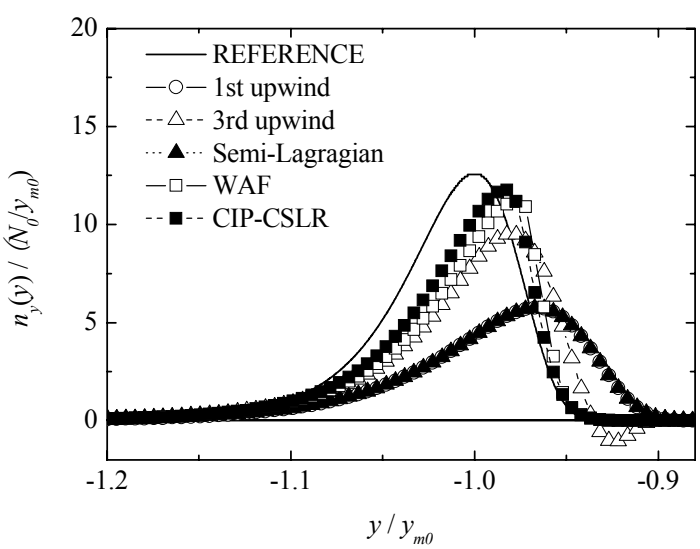

(b)

Fig. 2 Normalized particle number density function, $n_{y} /\left(N_{0} / y_{m 0}\right)$, at (a) $t / T_{c}=1.0$ and (b) $t / T_{c}=1.1$ in RUN-S4+CFL05.

\subsection{Numerical accuracy}

The L-2 error norm, $E_{r}$, is defined as

$$
E_{r}=\frac{1}{N_{\text {step }}}\left[\int\left(n_{y}-n_{y}{ }^{R E F}\right)^{2} d y / \int\left(n_{y}{ }^{R E F}\right)^{2} d y\right]^{1 / 2},
$$

where $n_{y}{ }^{R E F}$ is the reference value of $n_{y}$, which is the average between the WAF and CIP-CSLR results for RUN-REF, and $N_{\text {step }}$ the total number of time steps for computation.

Figure 3 shows $E_{r}$ at $t / T_{c}=1.0$ for RUN-CFL05. The gradient of each distribution indicates the spatial ( $y$-direction) accuracy. The first-order upwind scheme and first-order Semi-Lagrangian scheme have first-order accuracies and the others have second-order ones. The reason why the third-order upwind scheme has only second-order accuracy is that the splitting technique described in Equation (9) deteriorates the accuracy. Both the CIP-CSLR and WAF schemes have much less errors as well as second-order accuracies.

Figure 4 shows $E_{r}$ at $t / T_{c}=1.0$ for RUN-S4. The gradient indicates the temporal accuracy. All the schemes have first-order temporal accuracy, which comes from the first-order Euler scheme for time integrals. The important feature seen here is that the Semi-Lagrangian and CIP-CSLR schemes maintain first-order accuracy without collapsing even when $\alpha_{C F L}$ exceeds unity. This confirms that those Semi-Lagrangian based schemes are applicable to high-CFL computations, i.e., large-time-interval computations. The other 
schemes collapsed when $\alpha_{C F L}$ exceeded unity. The figure also clarifies that the CIP-CSLR scheme has a good feature of least errors.

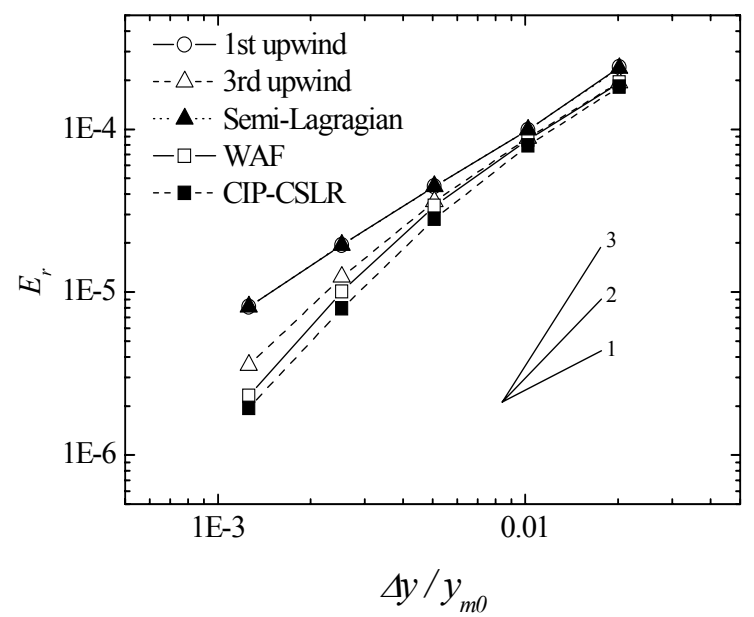

Fig. 3 L2 norms of errors, $E_{r}$, plotted against spatial ( $y$-direction) resolutions at $t / T_{c}=1.0$ in RUN-CFL05.

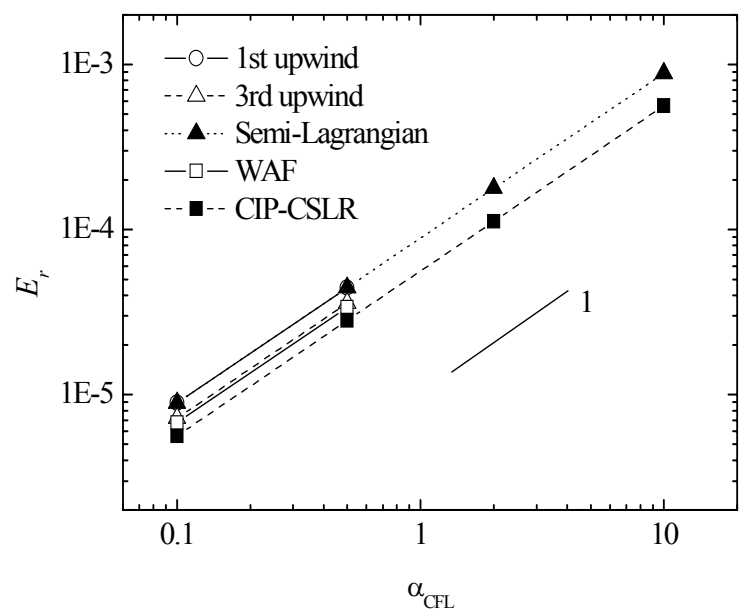

Fig. 4 L2 norms of errors, $E_{r}$, plotted against temporal resolutions at $t / T_{c}=1.0$ in RUN-S4.

\subsection{Conservation of particle numbers}

The number of droplets is changed when droplets evaporate or collide-coalesced. However, the total number is conserved while droplets are growing through condensation. The number conservation index, $\mathrm{CV}$, is defined to investigate the number conservation:

$$
C V=\left|\int n_{y}(y) d y-N_{0}\right| / N_{0} .
$$

During the condensation stage of $0<t / T_{c}<1.0, C V$ should be maintained at zero. Table 3 shows the values of $C V$ at $t / T_{c}=1.0$ for RUN-S4+CFL05. The third-order upwind scheme has two-order smaller value than the first-order schemes, however its value is not negligible. In contrast, the values from the WAF and CIP-CSLR schemes are in the order of $10^{-15}$, which are within the decimal errors. This confirms that the WAF and CIP-CSLR schemes are conservative schemes. 
Table 3 Number conservation index, $C V$, at $t / T_{c}=1.0$ in RUN-S4+CFL05.

\begin{tabular}{rc}
\hline advection scheme & $C V$ \\
\hline \hline 1st upwind & $1.48 \times 10^{-1}$ \\
\hline 3rd upwind & $4.07 \times 10^{-3}$ \\
\hline Semi-Lagragian & $1.47 \times 10^{-1}$ \\
\hline WAF & $1.39 \times 10^{-15}$ \\
\hline CIP-CSLR & $1.31 \times 10^{-15}$ \\
\hline
\end{tabular}

\subsection{Computational cost}

Table 4 shows the number of floating point operations (FPNO) for RUN-S4+CFL05. The values are normalized by the value of FPNO for the first-order upwind scheme. The CIP-CSLR scheme requires 3.3 times larger FPNO than the WAF scheme. This is, however, the result in the case of same time interval. As mentioned in Figure 4, the CIP-CSLR scheme is applicable to large-time-interval calculations, and therefore it is possible that the CIP-CSLR scheme achieves more economical calculation in total.

For example, Lynn et al. $(2005)^{(12)}$ used the time interval of $\Delta t=0.4 \mathrm{~s}$ for their spectral-bin model. They employed a Semi-Lagrangian scheme for phase change calculations. Therefore, the time interval was restricted not by a CFL restriction for phase change calculation, but by the coupling between phase change and temperature change or by the collision growth calculation. If they have employed one of the differential schemes for phase change calculation, the CFL restriction of $\alpha_{C F L}<1$ would have required much smaller time interval: Assuming the relative humidity of $99 \%(\sigma=-0.01)$ and temperature of $293 \mathrm{~K}$, the time interval should be set smaller than $2.1 \times 10^{-2} \mathrm{~s}$ to satisfy $\alpha_{C F L}<1$. This indicates that Lynn et al. (2005) could use 20 times larger time interval owing to the scheme applicable to high-CFL calculations than that restricted by the CFL.

Taking this fact into account, we can expect that the time interval for the CIP-CSLR scheme is more than 10 times larger than that used for the differential schemes such as the first-order and third-order upwind schemes and the WAF scheme. When we use 10 times larger time interval for the CIP-CSLR scheme, the total FPNO required becomes less than half of that for the first-order upwind scheme, and one-thirds for the WAF scheme. In this sense, the CIP-CSLR scheme is very economical in computational cost.

Table 4 Required number of floating-point-number operations (FPNO) for RUN-S4+CFL05. The number is normalized by that for the first-order upwind scheme. Asterisk $\left(^{*}\right)$ denotes the number when the CFL is set at 10 times larger than that in the first-order upwind scheme.

\begin{tabular}{rc}
\hline advection scheme & No. of FPNO \\
\hline \hline 1st upwind & 1.00 \\
\hline 3rd upwind & 1.31 \\
\hline Semi-Lagragian & $2.88\left(0.29^{*}\right)$ \\
\hline WAF & 1.34 \\
\hline CIP-CSLR & $4.39\left(0.44^{*}\right)$ \\
\hline
\end{tabular}

\section{Conclusions}

In spectral-bin models for cloud physics, water droplets are classified into tens or hundreds of size bins and number density functions are calculated for explicit consideration of droplet sizes. This study has introduced a new application of CIP-CSLR (the constrained 
interpolated profile-conservative Semi-Lagrangian with rational function) scheme for the calculation of droplet growth due to phase changes in the spectral-bin models. The droplet growth is treated as an advection of number densities between size bins, and the advection equation is solved using the CIP-CLSR scheme in our method.

In order to evaluate our method, we have performed an idealized numerical experiment for condensing and evaporating water droplets in a humid air. The results show that our CIP-CSLR-based method has second-order spatial accuracy and achieves number conservation during condensation growth. In order to investigate the relative features of our method, comparison runs have been conducted, where several conventional advection schemes were employed; first-order upwind scheme, third-order upwind scheme, first-order Semi-Lagrangian scheme and the weighted average flux (WAF) scheme with SUPERBEE-flux limiter. Comparisons have revealed the advantages of our method in numerical accuracy, numerical stability, number conservation and computational cost.

This study has focused on the use in cloud physics. The present scheme, however, will be applicable to engineering phenomena such as spray cooling and spray combustion.

\section{Acknowledgments References}

This research was partially supported by Core Research for Evolutional Science and Technology (CREST) Program "Advanced Model Development and Simulations for Disaster Countermeasures” of Japan Science and Technology Agency (JST).

\section{References}

(1) Reisner, R. et al., Explicit forecasting of supercooled liquid water in winter storms using the MM5 mesoscale model. Quarterly Journal of Royal Meteorological Society, Vol. 124 (1998), pp.1071-1107.

(2) Thompson, G. et al., Explicit forecasts of winter precipitation using an improved bulk microphysics scheme. Part I: Description and sensitivity analysis. Monthly Weather Review, Vol. 132 (2004), pp.519-542.

(3) Reisin, T. et al., Rain Production in Convective Clouds As Simulated in an Axisymmetric Model with Detailed Microphysics. Part I: Description of the Model, Journal of the Atmospheric Sciences, Vol. 53 (1996), pp. 497-519.

(4) Onishi, R. et al., Explicit droplet collisions in turbulent clouds in MSSG-A with a bin method, Proceeding of Third Asian-Pacific Congress on Computational Mechanics (APCOM07) in conjunction with Eleventh International Conference on Enhancement and Promotion of Computational Methods in Engineering and Sciences (EPMESC XI). (2007), CD-ROM.

(5) Toro, E.F., A weighted average flux method for hyperbolic conservation laws, Proceedings of the Royal Society of London, Vol. A423 (1989), pp.401-418.

(6) Lowe, D. et al., A condensed-mass advection based model for the simulation of liquid polar stratospheric clouds, Atmospheric Chemistry and Physics, Vol. 3 (2003), pp.29-38.

(7) Xiao, F. et al., Conservative and oscillation-less atmospheric transport schemes based on rational functions, Journal of Geophysical Research, Vol. 107 (2002), D22-4609.

(8) Soong, S., Numerical simulation of warm rain development in an axisymmetric cloud model. Journal of the Atmospheric Sciences, Vol. 31 (1974), pp.1262-1285.

(9) Berry, E.X., Cloud droplet growth by collection, Journal of the Atmospheric Sciences, Vol. 24 (1967), pp.688-701.

(10) Kawamura, T. et al., Computation of high Reynolds number flow around a circular cylinder with surface roughness, AIAA Paper, Vol. 84 (1984), pp.340.

(11) Tzivion, S. et al., Numerical simulation of hygroscopic seeding in a convective cloud, 
Journal of Applied Meteorology, Vol. 46 (1994), pp.252-27.

(12) Lynn, B.H. et al., Spectral (Bin) Microphysics Coupled with a Mesoscale Model (MM5). Part II: Simulation of a CaPE Rain Event with a Squall Line, Monthly Weather Review, Vol. 133 (2005), pp.59-71. 